\title{
Postdispersal Infection and Disease Development of Pyrenophora semeniperda in Bromus tectorum Seeds
}

\author{
Heather Finch-Boekweg, John S. Gardner, Phil S. Allen, and Brad Geary
}

First, third, and fourth authors: Brigham Young University, Department of Plant and Wildlife Sciences, Provo, UT 84602; and second author: Brigham Young University, Department of Biology, Provo, UT 84602. Accepted for publication 23 November 2015.

\begin{abstract}
Finch-Boekweg, H., Gardner, J. S., Allen, P. S., and Geary, B. 2016. Postdispersal infection and disease development of Pyrenophora semeniperda in Bromus tectorum seeds. Phytopathology 106:236-243.

The Ascomycete fungus, Pyrenophora semeniperda, attacks a broad range of cool-season grasses. While leaf and predispersal infection of seeds (i.e., florets containing caryopses) have been previously characterized, little is known about the pathogenesis of mature seeds following dispersal. In this study, we examined infection and disease development of $P$. semeniperda on dormant seeds of Bromus tectorum. Inoculated seeds were hydrated at $20^{\circ} \mathrm{C}$ for up to 28 days. Disease development was characterized using scanning electron and light microscopy. $P$. semeniperda conidia germinated on the seed surface within 5 to $8 \mathrm{~h}$. Hyphae grew on the seed surface and produced extracellular mucilage that eventually covered the seed. Appressoria

formed on the ends of hyphae and penetrated through the lemma and palea, stomatal openings, and broken trichomes. The fungus then catabolized the endosperm, resulting in a visible cavity by 8 days. Pathogenesis of the embryo was associated with progressive loss of cell integrity and proliferation of mycelium. Beginning at approximately day 11, one to several stromata (approximately $150 \mu \mathrm{m}$ in diameter and up to $4 \mathrm{~mm}$ in length) emerged through the lemma and palea. Degradation of embryo tissue was completed near 14 days. Conidiophores produced conidia between 21 and 28 days and often exhibited "Y-shaped" branching. This characterization of disease development corrects previous reports which concluded that $P$. semeniperda is only a weak seed pathogen with infection limited to the outermost seed tissues. In addition, the time required for disease development explains why infected dormant or slow-germinating seeds are most likely to experience mortality.
\end{abstract}

The plant pathogen, Pyrenophora semeniperda, which attacks a broad range of cool-season grasses, has primarily been studied for its role as a causal agent in foliar diseases of cereal crops (Medd et al. 2003). Generally observed in the anamorph state, Drechslera campanulata, P. semeniperda is reported to occur in North and South America, Australia, New Zealand, South Africa, and Eurasia (Shattuck 1993; Stewart et al. 2009; Yonow et al. 2004). Medd et al. (2003) presented a putative asexual life cycle for $P$. semeniperda that begins with infection of leaves and/or developing seeds and is completed with the production of conidiophores and conidia on stromata, as is typical for Ascomycetes. Because observations using light and scanning electron microscopy (SEM) failed to detect fungal hyphae in either the endosperm or embryo, Campbell and Medd (2003) concluded that infection was restricted to epidermal tissues in developing seeds. Based on laboratory observations that infected cereal crop seeds generally escape mortality and develop into normal seedlings, Campbell and Medd (2003) and Medd et al. (2003) concluded that postdispersal infection of mature seeds (caryopses) was possible, but likely to cause only a temporary reduction in seedling growth.

Several recent studies contradict the view that $P$. semeniperda acts as a weak seed pathogen. $P$. semeniperda can cause significant mortality to grass seeds infected following dispersal (Allen et al. 2013; Baughman and Meyer 2013; Beckstead et al. 2014; Meyer et al. 2008, 2014). For example, mature seeds of the winter annual species Bromus tectorum can suffer high mortality in field seed banks following exposure to soilborne $P$. semeniperda (Meyer et al. 2007).

B. tectorum is of particular importance in the western United States, where it has successfully invaded millions of hectares and

Corresponding author: P. S. Allen; E-mail address: phil_allen@byu.edu has become a weed of economic and ecological importance (DiTomaso 2000; Masi et al. 2014a). The species produces prolific amounts of seeds. In invaded ecosystems, it can yield over 30,000 seeds $\mathrm{m}^{-2}$ (Beckstead et al. 2010; Meyer et al. 2007). These seeds provide an abundant source of host material for $P$. semeniperda. Because seed mortality is easily determined (i.e., as indicated by the appearance of macroscopic stromata concurrent with failed seed germination as the end result of pathogenesis) (Finch et al. 2013), the $P$. semeniperda-B. tectorum pathosystem has recently received considerable attention as a model for studying seed pathogens in the soil (Allen et al. 2013; Beckstead et al. 2014; Meyer et al. 2014). Studies conducted pursuant to an understanding of this system have led to four general conclusions. First, slow-germinating or dormant seeds are most likely to suffer mortality from the fungus while fastgerminating seeds are likely to escape (Beckstead et al. 2007; Finch et al. 2013). Second, higher populations of the fungus occur at xeric rather than mesic sites due to lower autumn germination and subsequent secondary-dormancy induction in seeds (Allen et al. 2013; Hawkins et al. 2013; Meyer et al. 2014). Third, high levels of B. tectorum can promote spillover of $P$. semeniperda onto native grass communities (Beckstead et al. 2010). Fourth, the fungus can mediate competitive outcomes between seeds of B. tectorum and native grasses (Meyer et al. 2014).

While these studies clearly demonstrate that $P$. semeniperda can infect and kill mature seeds of B. tectorum and several other grasses under both laboratory and field conditions (Beckstead et al. 2014), details of seed pathogenesis leading to mortality have not been previously described. In this study, light microscopy and SEM were used to investigate the mode of attack of $P$. semeniperd $a$ on dormant $B$. tectorum seeds. In characterizing the process of infection and disease progression, we specifically sought to understand whether mycelium are restricted to outer seed layers as reported for developing seeds (Campbell and Medd 2003) or whether the endosperm and/or embryo are also colonized. 


\section{MATERIALS AND METHODS}

Seed population and fungal inoculum. Mature seeds of B. tectorum were collected from a wild population at the Brigham Young University Research Farm (Spanish Fork, Utah) in June 2011. Chaff was removed and seeds were stored in a $-10^{\circ} \mathrm{C}$ freezer to maintain dormancy. P. semeniperda was isolated from an infected B. tectorum seed found near Whiterocks, Utah, and inoculum of this fungus was produced on modified alphacel medium $\left(20^{\circ} \mathrm{C}, 12-\mathrm{h}\right.$ photoperiod) as described by Meyer et al. (2010). The isolate (WRK0) used in this study was a moderately virulent strain (Meyer et al. 2010).

Seed inoculation and sampling for SEM. Fifty seeds were inoculated with a single $P$. semeniperda conidium (low inoculation). An additional 50 seeds were inoculated with more than 100 conidia (high inoculation). Prior to inoculation, conidia were placed on a polycarbonate membrane (Poretics Corporation, Livermore, CA) and hydrated for $20 \mathrm{~min}$ with distilled water. In the lowinoculation treatment, an individual conidium was placed onto the lemma using a glass needle. Seeds were viewed under a dissecting microscope (Zeiss Axiovert 135; Carl Zeiss Microscopy, Jena, Germany) to ensure successful inoculation. Seeds receiving multiple conidia were inoculated by shaking them for $10 \mathrm{~s}$ in a vial with $3 \mathrm{mg}$ of conidia in $1 \mathrm{ml}$ of distilled water.

All inoculated seeds were placed on polycarbonate membranes laid on top of two water-saturated blue germination blotters (Anchor Paper, St. Paul, MN) inside a 100-mm, lid-enclosed Petri dish. (The polycarbonate membrane prevented seed contamination with fibers from the blue blotters, which interfered with SEM photography.) The Petri dishes were then placed in plastic sleeves and incubated on 12:12 h light/dark cycles with fluorescent lighting at $20^{\circ} \mathrm{C}$, which is within the reported optimum growth range for the pathogen (Campbell and Medd 2003). Incubating seeds were sampled at periods of $5,8,12,18$, and $24 \mathrm{~h}$ and $3,8,14,21$, and 28 days postinoculation.

Additional seed treatments. To independently verify that observed pathogenesis was due to $P$. semeniperda, at least 20 additional $B$. tectorum seeds inoculated with either 1 or $>100$ conidia were subjected to one of five pretreatments prior to inoculation, which were aimed to minimize possible contamination. These inoculation treatments included (i) removing the lemma and palea from each seed, (ii) autoclaving and then air drying seeds for $24 \mathrm{~h}$, (iii) short surface sterilizing by immersing seeds for $1 \mathrm{~min}$ in ethyl alcohol (70\%) followed by $1 \mathrm{~min}$ in a $10 \%$ bleach solution, or long sterilization by immersing seeds for 2 min in $70 \%$ ethyl alcohol followed by $2 \mathrm{~min}$ in $10 \%$ bleach, (iv) removal of the lemma and palea followed by either short or long surface sterilization as described, and (v) removal of the lemma and palea from each seed followed by autoclaving. Surfacesterilized seeds were rinsed with sterile distilled water before being inoculated.

Specimen preparation for SEM. Seeds were chemically fixed or freeze-dried following inoculation and incubation. Chemically fixed samples were placed directly in fixative $(2 \%$ glutaraldehyde solution buffered with sodium cacodylate at $\mathrm{pH} 7.3$ ) while freeze-dried seeds were frozen in liquid nitrogen, fractured into small pieces, and placed in $2 \%$ glutaraldehyde fixative. Following glutaraldehyde fixation, seeds were washed in cacodylate buffer, fixed and stained with $1 \%$ osmium tetraoxide solution

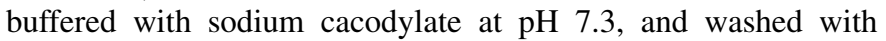
distilled water. Seeds were then dehydrated with a graded series of acetone, and then subjected to critical point drying. All samples were coated with gold/palladium and viewed with a scanning electron microscope (XL30 ESEM FEG, FEI Manufacturing, Hillsboro, OR).

Specimen preparation for sectioning and light microscopy. Seeds used for sectioning were inoculated with multiple conidia, and then incubated at $20^{\circ} \mathrm{C}$ as previously described for SEM.
Subsets of seeds were removed from Petri dishes on days 3, 8, or 14 and placed in liquid nitrogen for $20 \mathrm{~s}$. Seeds were fractured and fixed using procedures listed above for SEM preparation, but were not critically point dried. Following $\mathrm{OsO}_{4}$ fixation and staining, samples were embedded in Spurr's resin. Embedded samples were trimmed and sectioned approximately $1 \mu \mathrm{m}$ thick longitudinally or transversely using an 8-mm histological diamond knife (Electron Microscopy Sciences, Hatfield, PA). Samples were mounted on microscope slides and stained. The dye was a mixture of $0.25 \mathrm{~g}$ each of Borax, Toluidine Blue, and Azure II, with $25 \mathrm{ml}$ of distilled water. The mixture was stirred on a hotplate at low heat to $62^{\circ} \mathrm{C}$ for (approximately $10 \mathrm{~min}$ ) with a magnetic stir bar. When dry, samples were viewed with a light microscope for observation (Zeiss 4750039902, Bartles and Stout, Inc., Issaquah, WA).

\section{RESULTS}

Conidia of $P$. semeniperda were multiple-celled, oblong, and ranged from 30 to $100 \mu \mathrm{m}$ in length. Germination was most commonly observed from one or both polar cells (Fig. 1A), whether seeds were inoculated with one or multiple conidia. While germination from intermediate cells was not observed in our study, this has occasionally been observed with a light microscope (S. Clement, personal communication, 2013). Hydrated conidia germinated within 5 to $8 \mathrm{~h}$ of inoculation and produced germ tubes that developed into long, branched, slender hyphae (Fig. 1B), which gave rise to several penetration haustoria. Hyphae grew in multiple directions across the surface of the seed and produced extracellular mucilage within $12 \mathrm{~h}$. Both mycelium and associated mucilage spread across the seed over the course of disease development (Fig. 1C).

Hyphal penetration into the seed began within $24 \mathrm{~h}$ following inoculation. Appressoria formed on the seed surface at hyphal tips, and were club-shaped. Hyphae were observed to forcibly penetrate the lemma (Fig. 1D), palea, and stomata (Fig. 1E) but also penetrated the abscission layer and cracked surfaces in the lemma (Fig. 1F), palea, and trichomes (not shown). The ability of $P$. semeniperda hyphae to penetrate a variety of structures and openings suggests that seed entry is highly opportunistic. However, hyphae did not penetrate all available openings. We occasionally observed hyphae grow over open and closed stomata as well as broken trichomes. In addition, hyphae were observed to wrap around trichomes, providing further adhesion to the seed. Following penetration by the appressoria, mycelium were observed to exit and re-enter seeds (Fig. 1F), resulting in a visible network of mycelium on the surface.

Endosperm cross-sections showed progressive deterioration of tissue over time. Three days following inoculation, mycelium began to appear in the testa, but was not yet visible in the endosperm or aleurone cells (Fig. 2A and B). Starch granules and endosperm cell walls appeared intact. By 8 days, disease progression and associated catabolism of the endosperm led to the formation of a hollowed cavity that appeared to spread outward from the center of the endosperm (Fig. 2C and D). Lysis of cells was observed as an irregular but clearly delineated disease margin. Fourteen days following inoculation, mycelium was increasingly present within the remaining cells of the endosperm, and was also visible within the endosperm cavity (Fig. 2E). Stromata filled with visible, dense mycelium emerged from multiple locations around the seed (Fig. 2E and F).

Disease development in the embryo progressed more slowly than in the endosperm (Figs. 3 to 5). Three days following inoculation, embryonic cells within the scutellum, coleorhiza, coleoptile, radicle, and primordial root cap appeared free from visible signs of disease development (Fig. 3, insets 1 to 5) even though mycelium was visible within the testa (Fig. 2B) by this time. Cell walls were still intact and seed tissues were easily identified. Following 8 days of disease development, cell walls 
showed signs of deterioration, especially in the radicle (Fig. 4). In contrast with the endosperm, embryo cells were still distinguishable, but mycelium was visible throughout embryo tissues. Some cells had lysed by this time (Fig. 4, insets 1 to 5). Fourteen days following inoculation, mycelium had proliferated throughout the embryo (Fig. 5). Tissues were poorly defined as constituent cells were destroyed. After day 14, disease progression was sufficient such that sectioning seeds became very difficult and endosperm and embryo tissues were largely indistinguishable (data not shown). In addition, the blue dye used for staining was increasingly ineffective as cell features became indistinguishable.

Under the conditions of this study, most stromata emerged from seeds between 11 and 14 days following inoculation (Fig. 6). After this time, fewer new stromata emerged although stromata continued to elongate up to $>4 \mathrm{~mm}$ in length. Development of conidiophores followed stromata protrusion (Fig. 6A). When viewed 14 days following inoculation, stromata varied in height and conidiophores also varied in length (Fig. 6B). As stromata elongated, numerous conidiophores developed became more numerous (Fig. 6C). Prior to conidia formation, conidiophores occasionally showed alternating light-dark segmentation (Fig. 6D), although we are uncertain what this represents. We did not quantify conidia production. Only a few conidia were produced within 21 days following inoculation. Most conidia were observed between 21 and 28 days after inoculation. As conidia formed and dried, they appeared pinched at the attachment site of the conidiophore, and were most commonly observed to form in branched pairs (Fig. 6E). The appearance of black macroscopic stromata with no visible seed radicle indicated seed death (Fig. 6F).

Disease development in seeds subjected to additional treatments (i.e., lemma and palea removal, autoclaving, and surface-sterilization) all showed a similar pattern to that of continuously hydrated dormant seeds. Lemma and palea removal and surface-sterilization accelerated disease, presumably due to easier entry of hyphae into the seed following these treatments. All autoclaved seeds were killed. This treatment resulted in dramatic acceleration of disease development. In this case, the fungus produced stromata within 5 to 6 days (not shown).

\section{DISCUSSION}

Dormant $B$. tectorum seeds exhibit the capability to become readily infected by $P$. semeniperda, ultimately resulting in seed mortality. In contrast with previous reports that this fungus is a weak seed pathogen and only infects epidermal layers in seeds (Campbell and Medd 2003; Medd and Campbell 2005), our observations clarify that the fungus can penetrate and grow throughout the seed. While living seed tissues appear to delay disease progression, all major seed components are ultimately vulnerable to $P$. semeniperda.

Conidia of $P$. semeniperda germinated on seeds beginning $5 \mathrm{~h}$ following the onset of imbibition. The same isolate used in this study germinated in water and potato dextrose agar (PDA) in as little as $2 \mathrm{~h}(\mathrm{H}$. Finch-Boekweg, unpublished data). More time may be required for conidia germination on seeds because water must reach conidia by first passing through or around the seed. We have thus far found no evidence that $P$. semeniperda conidia germination requires or is improved by seed exudates as has been reported for numerous fungal species (Nelson 2004). Conidia of P. semeniperda have previously been reported to germinate within $3 \mathrm{~h}$ following the onset of imbibition (Campbell and Medd 2003). Conidia from 43 isolates
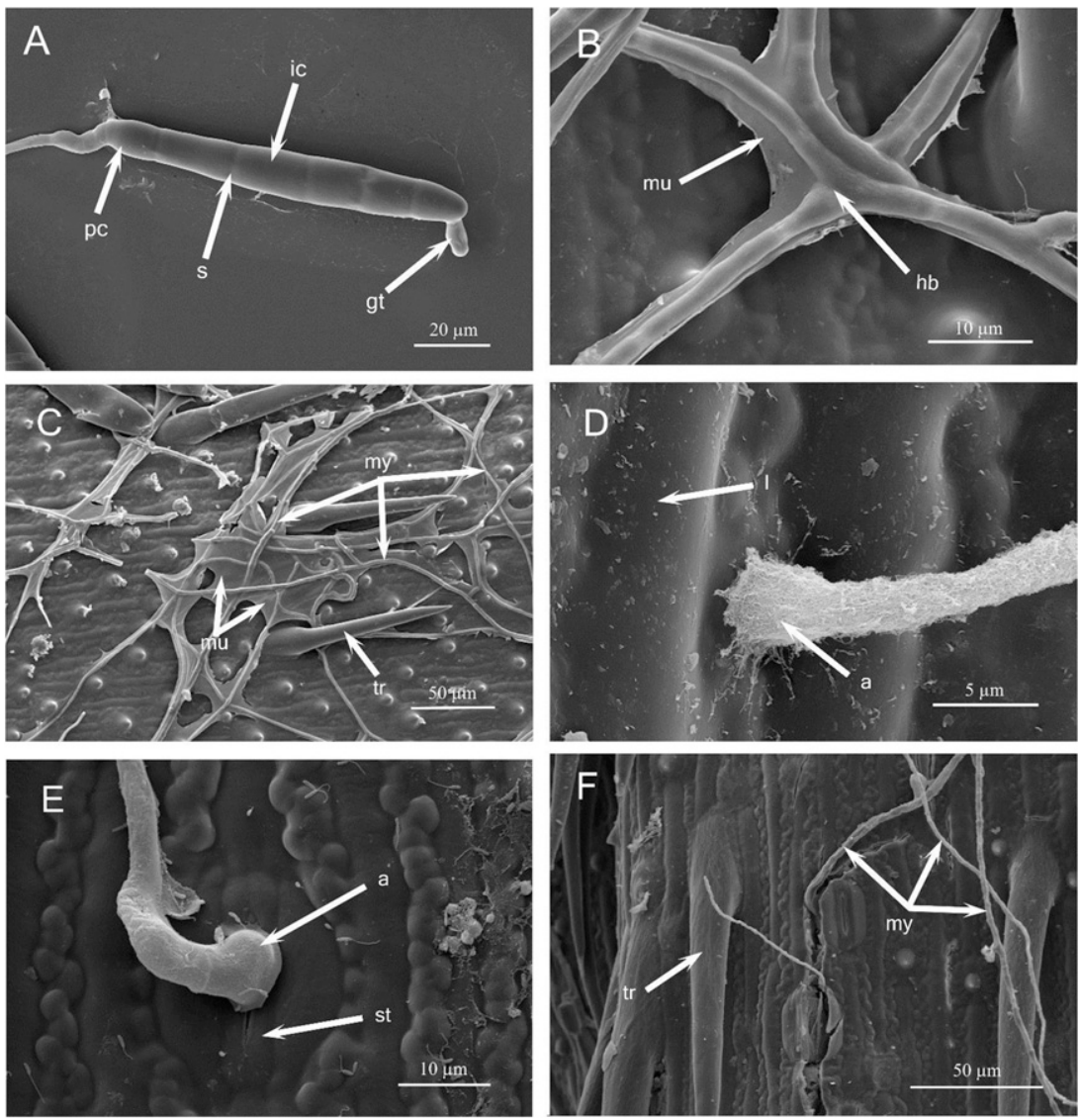

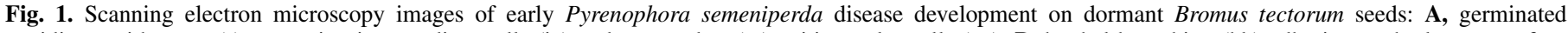

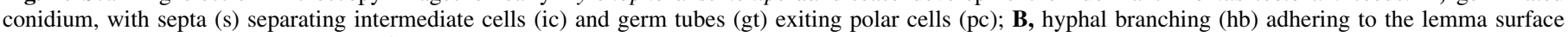

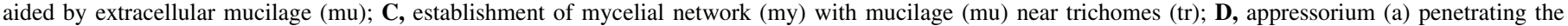
lemma (1); E, appressorium (a) penetrating a stomata (st); and F, mycelium (my) entering and exiting a crack in the lemma between trichomes (tr). 

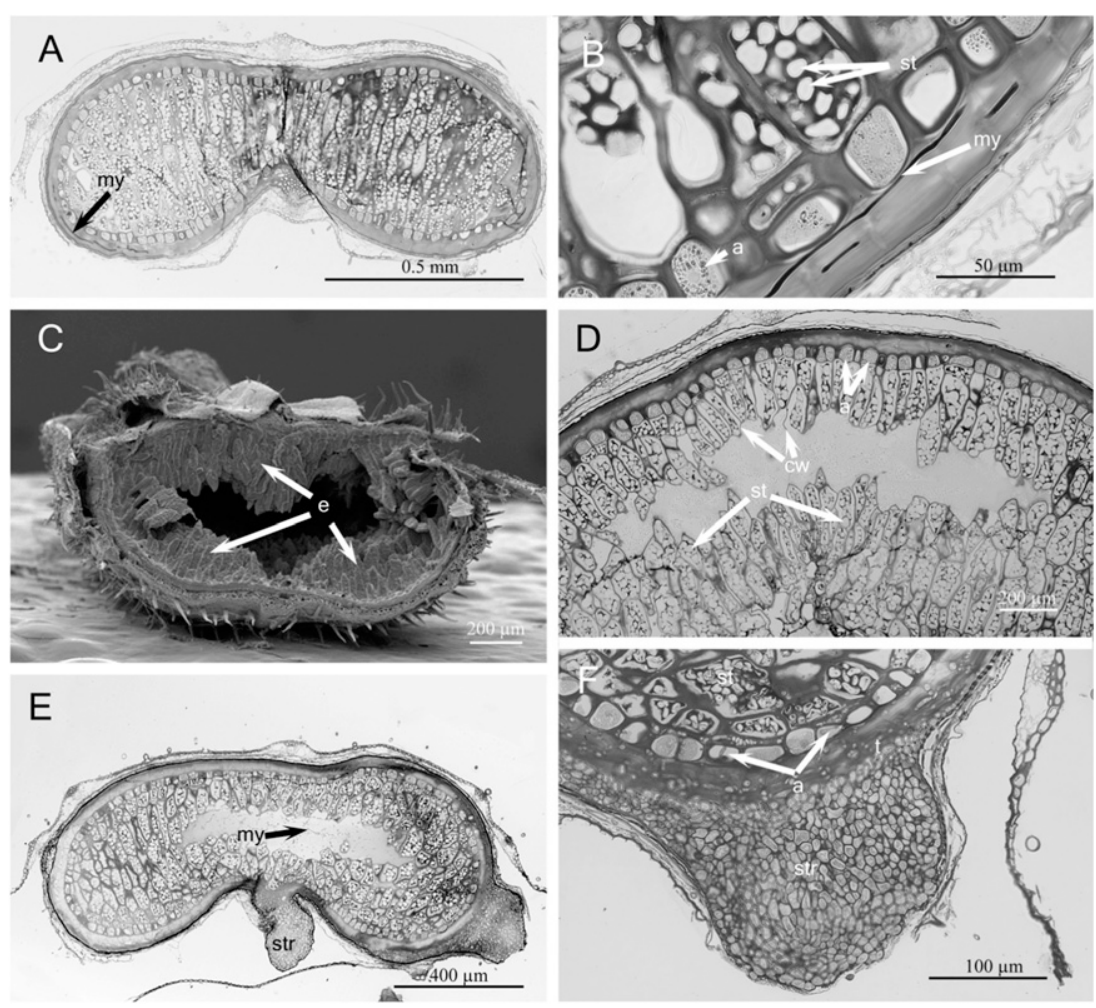

Fig. 2. Disease development of Pyrenophora semeniperda in the endosperm of dormant Bromus tectorum seeds after 3 days (A and B), 8 days (C and D), or 14 days ( $\mathbf{E}$ and $\mathbf{F})$. All images are cross sections taken near the middle of the seed, and all are light micrographs except image $\mathrm{C}$, which is scanning electron microscopy: A, largely intact endosperm cells with mycelium (my) beginning to appear within the testa; $\mathbf{B}$, enlarged view showing intact aleurone cells (a), starch granules (st), and darkly stained mycelium (my) growing in the testa; C, pathogenesis of the endosperm (e) spreads from the center as indicated by open cavity; D, lysis of starch granules (st) and cell walls (cw) associated with tissue breakdown as the endosperm cavity enlarges. The living aleurone cells (a) are still largely intact; E, further pathogenesis of the endosperm with some mycelium (my) visible in central cavity and a stroma (str) emerging from the ventral side; and F, enlarged view of fungal stroma (str) from the seed, with the testa ( $t$ ), aleurone cells (a), and remaining starch granules (st).

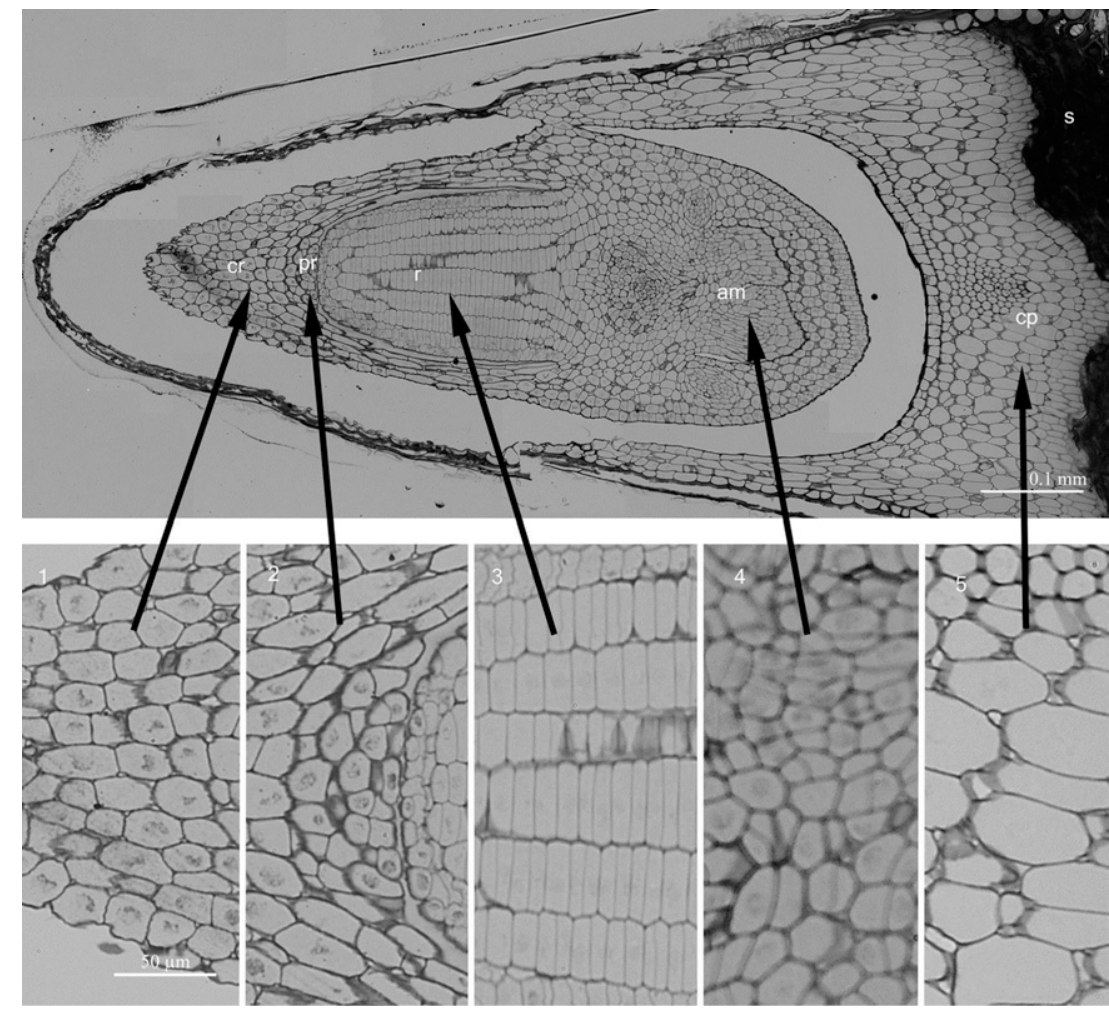

Fig. 3. Longitudinal sections of a hydrated Bromus tectorum seed 3 days following inoculation with Pyrenophora semeniperda conidia. Embryonic tissues lack visible disease development. Top micrograph shows coleorhiza (cr), primordial rootcap (pr), radicle (r), apical meristem (a), coleoptile (cp), and scutellum (s). Lower insets 1 to 5 are enlargements of areas indicated by the black arrows ( $50 \mu \mathrm{m}$ bar applies to all). 


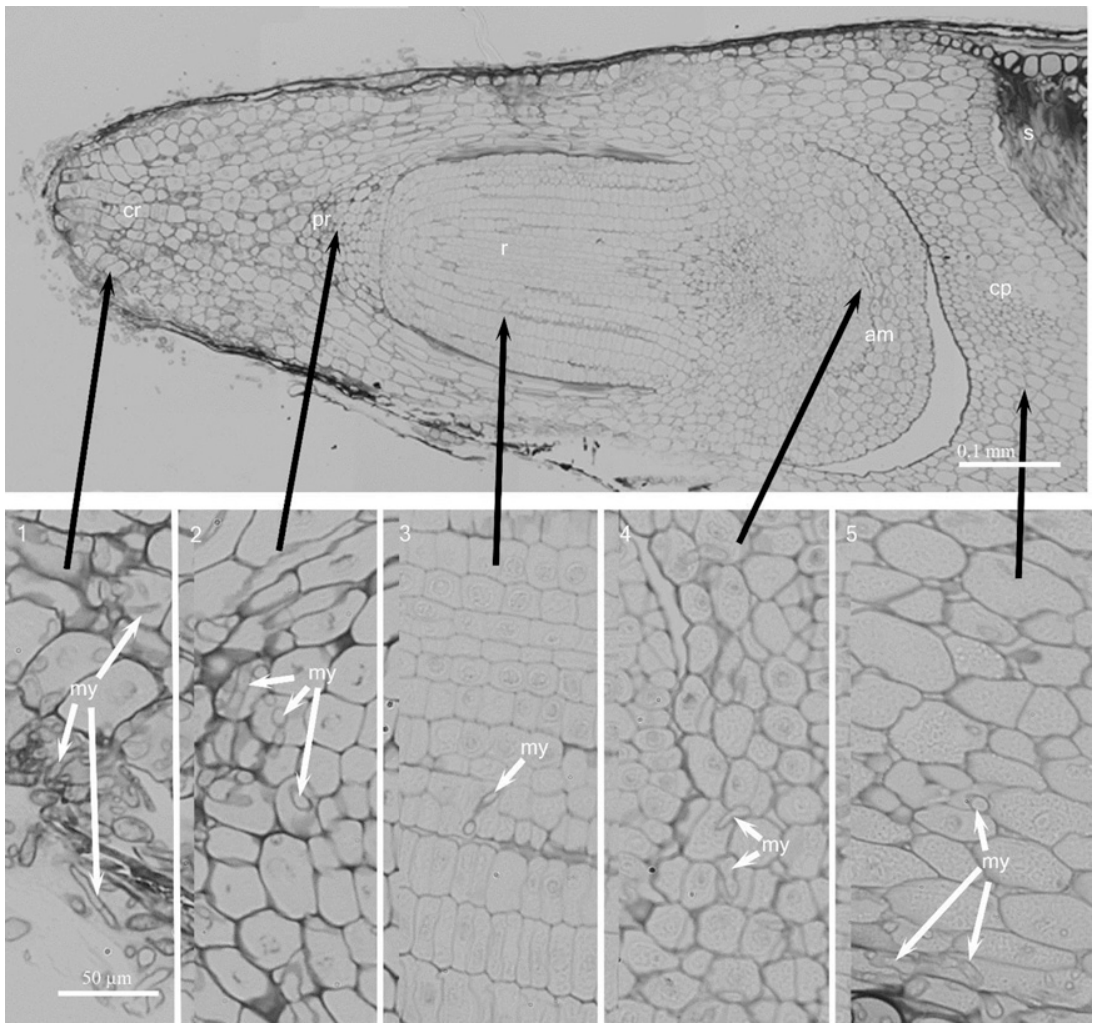

Fig. 4. Longitudinal sections of a hydrated Bromus tectorum seed 8 days following inoculation with Pyrenophora semeniperda conidia. Embryonic tissues show visible disease development as indicated by cell lysis and the presence of mycelium. Top micrograph shows coleorhiza (cr), primordial rootcap (pr), radicle (r), apical meristem (am), coleoptile (cp), and scutellum (s). Lower insets 1 to 5 are enlargements of areas indicated by the black arrows. White arrows highlight representative fungal mycelium (my) in each tissue.

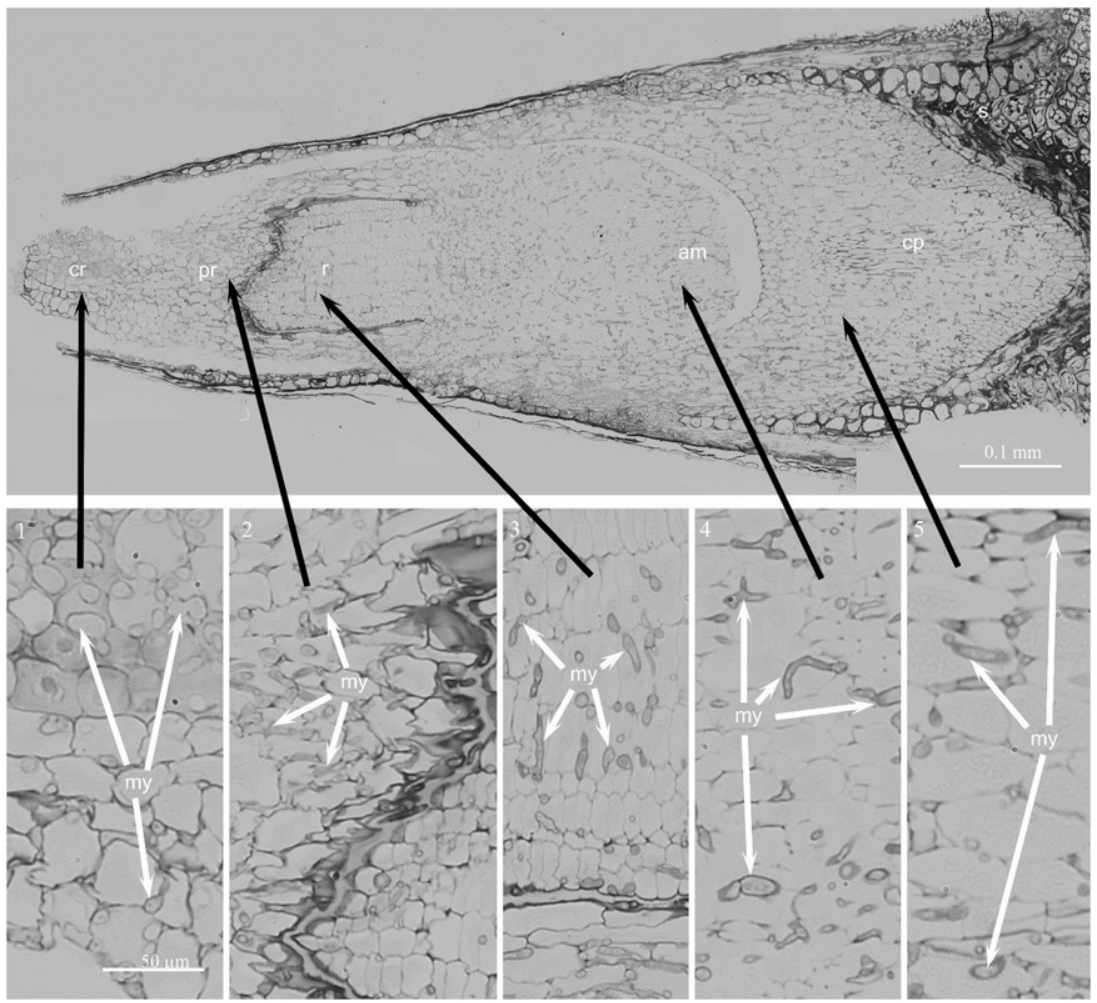

Fig. 5. Longitudinal sections of a hydrated Bromus tectorum seed 14 days following inoculation with Pyrenophora semeniperda conidia. Embryonic tissues show advanced disease development as indicated by a high loss of cell structure and proliferation of mycelium. Top micrograph shows coleorhiza (cr), primordial rootcap (pr), radicle (r), apical meristem (a), coleoptile (cp), and scutellum (s). Lower insets 1 to 5 are enlargements of areas indicated by the black arrows. White arrows highlight representative fungal mycelium (my) in each tissue. 
used in virulence trials germinated to nearly $100 \%$ within $6 \mathrm{~h}$ on PDA (Meyer et al. 2010). The rapid germination of conidia observed in this and other recent studies (Barth et al. 2015) suggests that dormancy is absent in conidia of this fungus.

In the present study, P. semeniperda germ tubes were observed to arise from one or both polar cells within $5 \mathrm{~h}$ of inoculation. For related Ascomycete species, germ tubes have been observed to grow from either polar or intermediate cells, but they have never been reported to germinate from both simultaneously (Arora et al. 1980; Coyle and Cooke 1993; Cromey and Cole 1985).

Under the near-ideal growing conditions for $P$. semeniperda that we used in this study, mycelium were just beginning to appear in the testa 3 days following inoculation and seed imbibition. The endosperm and embryo failed to show significant disease progression at this time. Under similar incubation conditions $\left(20^{\circ} \mathrm{C}, 12-\mathrm{h}\right.$ photoperiod), populations of nondormant $B$. tectorum seeds showed near-complete germination (radicle emergence) within 2 to 4 days (Allen et al. 1995; Beckstead et al. 2007). Rapid germination allows seeds to escape $P$. semeniperda-induced mortality even when seed infection has occurred. In many cases, stromatal production can be preceded by radicle emergence with seedlings that develop into normal plants (Beckstead et al. 2014; Medd et al. 2003). The observation that $P$. semeniperda often fails to kill infected $B$. tectorum seeds is restricted to rapidly germinating, nondormant seeds (Beckstead et al. 2007). P. semeniperda clearly kills dormant seeds and infects all major seed tissues.

In experiments using both natural and artificial inoculation of $B$. tectorum seeds of varying germination rates (Beckstead et al. 2007), P semeniperda killed seeds that germinated slowly while seeds that germinated quickly usually escaped. This result led
Beckstead and others (2007) to conclude that the fungus and seed (i.e., embryo) are in a "race" for endosperm resources. Earlier studies document the importance of relative growth rates on the outcome of fungal-seed interactions. For example, in experiments using seeds of five crop species and four seed/seedling pathogens, Leach (1947) clearly demonstrated that altering growth rates through temperature manipulations could profoundly influence the severity of damping off. In the $P$. semeniperda-B. tectorum pathosystem, a variety of environmental conditions that lead to slow germination can dramatically increase seed mortality, including incubation at reduced water potentials (Finch et al. 2013), hydration-dehydration cycles (Finch 2013), incubation at low temperatures coupled with intermediate water potentials and incubation at temperatures that favor growth of the fungus relative to seed germination of the host (Hawkins 2014).

As hyphae developed into a mycelial network (Harris 2008), a mucilaginous matrix was observed to coat much of the seed surface. The role of fungal mucilage in host recognition, adhesion, and penetration has been well documented (Epstein and Nicholson 2006; Howard et al. 1991; Jones and Epstein 1989; Tucker and Talbot 2001). We also observed hyphae wrapping around trichomes, which favors adhesion as reported in studies reviewed by Tucker and Talbot (2001). In our study, P. semeniperda penetrated a variety of structures and openings, but passed over some openings as has previously been reported (Babu et al. 2007; Campbell and Medd 2003; Wong et al. 2012).

Our observations of the formation and penetration of appressoria on mature $B$. tectorum seeds confirm previously reported results for $P$. semeniperda penetration into developing wheat seeds (Campbell and Medd 2003). In both cases, appressoria developed directly over
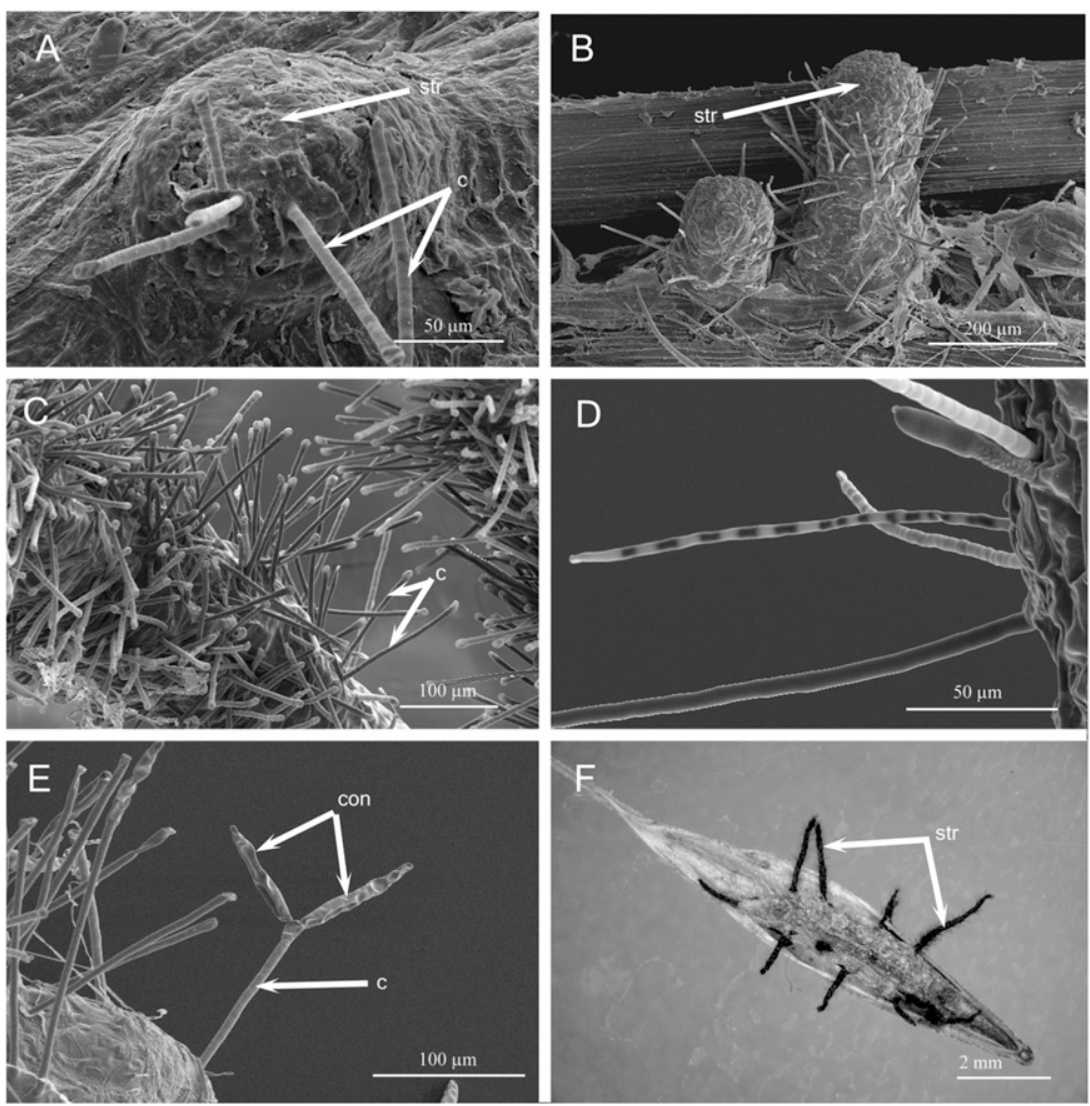

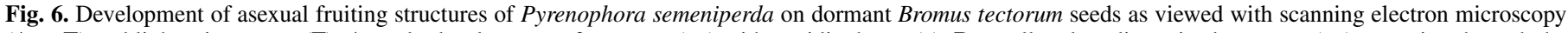

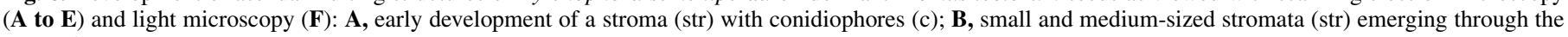

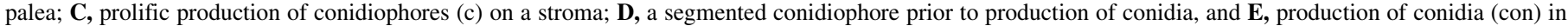
pairs on a conidiophore (c) 21 days following inoculation; F, a seed with macroscopic stromata (str) that indicate seed mortality. 
cell wall junctions, stomatal guard cells, and trichomes. In addition, we observed appressoria formation over the abscission layer. Regardless of tissue being penetrated, appressoria were generally observed $24 \mathrm{~h}$ following inoculation and imbibition. However, based on light microscopy and SEM observations, (Campbell and Medd 2003) failed to observe hyphae in the endosperm or embryo tissues of developing wheat seeds, and concluded that infection hyphae were confined to the epidermal layers. This contrasts with our observations that disease development progresses throughout the entire seed.

Infection of B. tectorum seeds generally occurred within $24 \mathrm{~h}$ following inoculation and incubation. In a related study investigating the effects of seed dehydration following infection, seed mortality was much more likely when inoculated seeds were imbibed for a period of $24 \mathrm{~h}$ prior to dehydration (Finch 2013). Inoculated seeds imbibed for only $8 \mathrm{~h}$ generally escaped mortality following subsequent dehydration-rehydration. Medd et al. (2003) concluded that $P$. semeniperda survives across generations as mycelium in grass seeds. Stromata later emerge from these seeds during germination. These findings combined suggest that mycelium exhibit desiccation tolerance once they successfully penetrate the seed, allowing disease development to continue when seeds are rehydrated.

Observations with SEM indicated that appressorial penetration occurred within $24 \mathrm{~h}$. Within 8 days following inoculation, the endosperm center began to hollow and cells and starch granules were completely missing. Living cells within the embryo and aleurone layer were then catabolized, which indicates late-stage disease development. Within 11 to 14 days following inoculation, macroscopic stromata emerged from the dormant, continuously hydrated seeds. SEM images did not clarify whether the lemma and palea were catabolized or if emerging stromata ruptured these tissues.

We observed that $P$. semeniperda completed the disease cycle more rapidly in autoclaved seeds, which produced stromata in as little as 5 days. This suggests that living mature seeds have defenses that delay disease development or, alternatively, that autoclaving resulted in physical damage to seeds and allowed for more rapid disease development.

Similar to its necrotrophic relatives which cause foliar diseases of cereal crops (e.g., P. tritici-repentis, P. teres), P. semeniperda secretes toxins creating a front of dead tissue that is digested enzymatically to provide nutrition (Meyer et al. 2010). P. semeniperda has been reported to produce a number of phytotoxins including cytochalasin B, a secondary metabolite that interferes with cytokinesis (Masi et al. 2014a, b, c). Roles for other phytotoxins produced by this fungus are less understood at present. Dormant seeds rely on physical and chemical defense mechanisms to exclude predators and pathogens (Dalling et al. 2011). In some cases, plants attacked by pathogens produced seeds containing higher concentrations of defense compounds compared with control plants that were not infected (Holeski et al. 2012; Shattuck 1993). However, such defenses appear to be absent in the P. semeniperda-B. tectorum pathosystem.

Medd et al. (2003) presented and discussed a putative disease cycle for $P$. semeniperda. They suggested that conidia disseminate to the inflorescence and directly infect developing ovaries in grass hosts during anthesis. The fungus then passes through the dry seed state as mycelium in grass seeds. The mycelia ultimately develop stromata that produce conidia borne on conidiophores. The present study, along with several recent studies cited herein, provides evidence that postdispersal inoculation and infection of mature seeds should be added to our understanding of the disease cycle of this fungus. Furthermore, because significant seed mortality following infection and disease progression occurs, especially under conditions that result in slow seed germination, $P$. semeniperda should be viewed as a potentially significant seed pathogen.

\section{ACKNOWLEDGMENTS}

This research was funded by the U.S. Department of Agriculture Joint Fire Sciences Program (JFSP-2011-S-2-6). We thank M. Nielson and J. Ryan Stewart for careful review and helpful suggestions in the preparation of this manuscript.

\section{LITERATURE CITED}

Allen, P. S., Meyer, S. E., and Beckstead, J. 1995. Patterns of seed afterripening in Bromus tectorum L. J. Exp. Bot. 46:1737-1744.

Allen, P. S., Meyer, S. E., and Beckstead, J. 2013. A predictive model for soil seedbank outcomes in the Pyrenophora semeniperda-Bromus tectorum pathosystem. Plant Prot. Sci. 49(S):21-23.

Arora, R. K., Mandahar, C. L., and Pahwa, R. K. 1980. Infection of oat leaves by Helminthosporium avenae I. Infection process. Indian J. Mycol. Plant Pathol. 10:8-11.

Babu, A. M., Philip, T., and Kumar, V. 2007. Development of the leaf spot fungus, Cercospora ricinella, on castor leaf-An SEM account. J. Phytopathol. 155:426-430.

Barth, C. W., Meyer, S. E., Beckstead, J., and Allen, P. S. 2015. Hydrothermal time models for conidial germination and mycelial growth of the seed pathogen Pyrenophora semeniperda. Fungal Biol. 119:720-730.

Baughman, O. W., and Meyer, S. E. 2013. Is Pyrenophora semeniperda the cause of downy brome (Bromus tectorum) die-offs? Invasive Plant Sci. Manage. 6:105-111.

Beckstead, J., Meyer, S. E., Connolly, B. M., Huck, M. B., and Street, L. E. 2010. Cheatgrass facilitates spillover of a seed bank pathogen onto native grass species. J. Ecol. 98:168-177.

Beckstead, J., Meyer, S. E., Molder, C. J., and Smith, C. 2007. A race for survival: Can Bromus tectorum seeds escape Pyrenophora semeniperdacaused mortality by germinating quickly? Ann. Bot. (Lond.) 99:907914.

Beckstead, J., Meyer, S. E., Reinhart, K. O., Bergen, K. M., Holden, R., and Boekweg, H. F. 2014. Factors affecting host range in a generalist seed pathogen of semi-arid shrublands. J. Plant Ecol. 215:427-440.

Campbell, M. A., and Medd, R. W. 2003. Leaf, floret and seed infection of wheat by Pyrenophora semeniperda. Plant Pathol. 52:437-447.

Coyle, P. M., and Cooke, B. M. 1993. Scanning electron micrographs of barley leaves infected with Drechslera teres. Mycologist 7:109-111.

Cromey, M. G., and Cole, A. L. J. 1985. Cytology of the host-pathogen interactions between Lolium perenne and Drechslera dictyoides. Plant Pathol. 34:83-94.

Dalling, J. W., Davis, A. S., Schutte, B. J., and Elizabeth Arnold, A. 2011. Seed survival in soil: Interacting effects of predation, dormancy and the soil microbial community. J. Ecol. 99:89-95.

DiTomaso, J. M. 2000. Invasive weeds in rangelands: Species, impacts, and management. Weed Sci. 48:255-265.

Epstein, L., and Nicholson, R. L. 2006. Adhesion and adhesives of fungi and oomycetes. Pages 41-62 in: Biological Adhesives. A. J. Smith and J. A. Callow, eds. Springer, Berlin.

Finch, H. 2013. The Pyrenophora semeniperda-Bromus tectorum pathosystem. Thesis. Brigham Young University, Provo, UT.

Finch, H., Allen, P. S., and Meyer, S. E. 2013. Environmental factors influencing Pyrenophora semeniperda-caused seed mortality in Bromus tectorum. Seed Sci. Res. 23:57-66.

Harris, S. B. 2008. Branching of fungal hyphae: Regulation, mechanisms and comparison with other branching systems. Mycologia 100:823-832.

Hawkins, K. 2014. secondary dormancy and summer conditions influence outcomes in the Pyrenophora semeniperda-Bromus tectorum pathosystem. Thesis. Brigham Young University, Provo, UT.

Hawkins, K., Allen, P. S., and Meyer, S. E. 2013. Secondary seed dormancy of seeds in relation to the Pyrenophora seminiperda-Bromus tectorum pathosystem. Plant Prot. Sci. 49(S):10-14.

Holeski, L. M., Jander, G., and Agrawal, A. A. 2012. Transgenerational defense induction and epigenetic inheritance in plants. Trends Ecol. Evol. 27: 618-626.

Howard, R. J., Ferrari, M. A., Roach, D. H., and Money, N. P. 1991. Penetration of hard substrates by a fungus employing enormous turgor pressures. Proc. Natl. Acad. Sci. USA 88:11281-11284.

Jones, M. J., and Epstein, L. 1989. Adhesion of Nectria haematococca macroconidia. Physiol. Mol. Plant Pathol. 35:453-461.

Leach, L. D. 1947. Growth rates of host pathogen as factors determining the severity of preemergence damping-off. J. Agric. Res. 75:161-179.

Masi, M., Evidente, A., Meyer, S. E., Nicholson, J., and Munoz, A. 2014a. Effect of strain and cultural conditions on the production of cytochalasin B by the potential mycoherbicide Pyrenophora semeniperda (Pleosporaceae, Pleosporales). Biocontrol Sci. Technol. 24:53-64. 
Masi, M., Meyer, S. E., Cimmino, A., Andolfi, A., and Evidente, A. 2014b. Pyrenophoric acid, a phytotoxic sesquiterpenoid penta-2,4-dienoic acid produced by a potential mycoherbicide, Pyrenophora semeniperda. J. Nat. Prod. 77:925-930.

Masi, M., Meyer, S. E., Clement, S., Andolfi, A., Cimmino, A., and Evidente, A. 2014c. Spirostaphylotrichin W, a spirocyclic $\gamma$-lactam isolated from liquid culture of Pyrenophora semeniperda, a potential mycoherbicide for cheatgrass (Bromus tectorum) biocontrol. Tetrahedron 70:1497-1501.

Medd, R. W., and Campbell, M. A. 2005. Grass seed infection following inundation with Pyrenophora semeniperda. Biocontrol Sci. Technol. 15:21-36.

Medd, R. W., Murray, G. M., and Pickering, D. I. 2003. Review of the epidemiology and economic importance of Pyrenophora semeniperda. Australas. Plant Pathol. 32:539-550.

Meyer, S. E., Beckstead, J., Allen, P. S., and Smith, D. C. 2008. A seed bank pathogen causes seedborne disease: Pyrenophora semeniperda on undispersed grass seeds in western North America. Can. J. Plant Pathol. 30: 525-533.

Meyer, S. E., Merrill, K. T., Allen, P. S., Beckstead, J., and Norte, A. S. 2014. Indirect effects of an invasive annual grass on seed fates of two native perennial grass species. Oecologia 174:1401-1413.
Meyer, S. E., Quinney, D., Nelson, D. L., and Weaver, J. 2007. Impact of the pathogen Pyrenophora semeniperda on Bromus tectorum seed bank dynamics in North American cold deserts. Weed Res. 47:54-62.

Meyer, S. E., Stewart, T. S., and Clement, S. 2010. The quick and the deadly: Growth vs. virulence in a seed bank pathogen. New Phytol. 187:209-216.

Nelson, E. B. 2004. Microbial dynamics and interactions in the spermosphere. Annu. Rev. Phytopathol. 42:271-309.

Shattuck, V. I. 1993. Glucosinolates and glucosinolate degradation in seeds from turnip mosaic virus-infected rapid cycle Brassica campestris L. plants. J. Exp. Bot. 44:963-970.

Stewart, T. E., Allen, P. S., and Meyer, S. E. 2009. First report of Pyrenophora semeniperda in Turkey and Greece. Plant Dis. 93:1351.

Tucker, S. L., and Talbot, N. J. 2001. Surface attachment and pre-penetration stage development by plant pathogenic fungi. Annu. Rev. Phytopathol. 39: 385-417.

Wong, M. H., Henderson, J., Aitken, E. A., and Drenth, A. 2012. Mode of infection of Phyllosticta maculata on banana as revealed by scanning electron microscopy. J. Phytopathol. 161:135-141.

Yonow, T., Kriticos, D. J., and Medd, R. W. 2004. The potential geographic range of Pyrenophora semeniperda. Phytopathology 94:805-812. 